\title{
Thank you to our Reviewers
}

We are indebted to the expert referees who have reviewed submissions to the Canadian Journal of Neurological Sciences in 2013 (names in bold reviewed five or more papers). Their thoughtfulness and expertise have served our journal well.

\begin{tabular}{|c|c|c|c|c|}
\hline Abdollah, Hoshiar & Camicioli, Richard & Dowling, James & Grover, Kavita M. & Katzberg, Hans D. \\
\hline Abuzinadah, Ahmad R. & Campbell, Craig & Duff Canning, Sarah & Guerra, Gonzalo G. & Kaufmann, Anthony M. \\
\hline Adatia, Sweta & Carter, Sherri L. & Easton, Alexander & Guzman, Juan & Keith, Julia L. \\
\hline Agid, Ronit & Casaubon, Leanne K. & Elbers, Jorina & Haddara, Wael M.R. & Kestle, John \\
\hline Ahmed, S. Nizam & Cendes, Iscia Lopes & Falero, Raul & Hadjivassiliou, M. & Khandji, Alexander \\
\hline Alhazzaa, Mohammed & Chan, Ming & Findlay, J. Max & Hamilton, Mark & Kirton, Adam \\
\hline Alikhani, Katayoun & Chan, Richard K.T. & Finestone, Hillel M. & Hanlon, Colleen & Klawiter, Eric C. \\
\hline Alturkustani, Murad & Chau, Vann & Finger, Elizabeth & Harris, Ashley D. & Korinthenberg, Rudolf \\
\hline Andermann, Frederick & Chayer, Celine & Fisher, Barbara J. & Haugarvoll, Kristoffer & Korngut, Lawrence \\
\hline Andrade, Danielle M. & Che, Jingjin & Fisk, John D. & Hayhurst, Caroline & Koski, Lisa \\
\hline Arjona, Antonio & Chow, Michael & Fourney, Daryl R. & Hogan, David B. & Kraus, Jorg \\
\hline Baker, Glen & Christie, Sean & Fournier, Christina & Hogan, Matthew J. & Krieger, Charles \\
\hline Baker, Steven K. & Chu, Joseph Y. & Fox, Richard J. & Horvath, Gabriella A. & Krings, Timo \\
\hline Bassani, Roberto & Chudley, Albert & Fox, Susan H. & Houlden, David & Kurtuncu, Murat \\
\hline Bauman, Glenn S. & Cooke, Lara J. & Fraser, Alex & Howse, David & Kurzepa, Jacek \\
\hline Becker, Werner J. & Cooper, Paul E. & Freedman, Mark S. & Hsiung, Ging-Yuek R. & Lafontaine, Anne- \\
\hline Benassi, Barbara & Cossette, Patrick & Freedman, Morris & Hsiung, Robin & Louise \\
\hline Benstead, Timothy & Costello, Fiona & Friedman, Jan & Hu, Xueqiang & Lagae, Lieven \\
\hline Berbrayer, David & Cote, Robert & Frosini, Maria & Hukin, Juliette & Lang, Anthony E. \\
\hline Bharatha, Aditya & Coutts, Shelagh & Furtado, Sarah & Huot, Philippe & Larue, Sandrine \\
\hline Bharati, Sachidanand J. & Crossley, Margaret & Gagnon, Cynthia & Hussein, Shazam & Lazosky, Andrea \\
\hline Bouchard, Manon & DeMeirleir, Linda & Ginsberg, H.J. & Jia, Jianhang & Lin, Lian-Feng \\
\hline Boulanger, Jean-Martin & Demos, Michelle & Glass, Jonathan & Jia, Longfei & Loewenstein, David \\
\hline Brais, Bernard & Desbiens, Richard & Gofton, Teneille & Jichici, Draga & Longman, Stewart \\
\hline Bramall, Alexa N. & Deschaintre, Yan & Goobie, Sharan & Jickling, Glen C. & Loock, Christine \\
\hline Briemberg, Hannah & Dhar, Rajat & Goodridge, Alan & Jog, Mandar S. & Lu, Zhengqi \\
\hline Bril, Vera & Di Legge, Silvia & Goodyear, Bradley G. & Johnson, Edward S. & Macaulay, Robert \\
\hline Brown, Authur & Dilli, Esma & Goyal, Mayank & Johnston, Janine L. & MacDonald, David B. \\
\hline Brown, W.F. & Diosy, David C. & Graham, Brett & Jones-Gotman, M. & Macdonald, Loch \\
\hline Brunet, Donald G. & Dodig, Dubravka M. & Graham, Candida & Jutras-Aswad, Didier & MacKnight, Chris \\
\hline Bullock, Martin & Doherty, Tim & Graham, Simon & Karamchandani, Jason & Maguire, John A.C. \\
\hline Burneo, Jorge G. & Donner, Elizabeth & Grant, Ian & Karim, Aftab S. & Mandell, Daniel M. \\
\hline Burton, Jodie M. & Dooley, Joseph M. & Griesdale, Donald E.G. & Kassel, Edward & Mandzia, Jennifer \\
\hline Busche, Kevin & dos Santos, Marlise P. & Grimes, David A. & Kate, Mahesh & Maranda, Bruno \\
\hline
\end{tabular}




\begin{tabular}{|c|c|c|c|c|}
\hline Marrie, Ruth Ann & Murtaza, Muhammed & Rajput, Alex & Spears, Julian & Veilleux, Martin \\
\hline Marriott, James J. & Myles, S. Terence & Rana, Abdul Q. & Spence, J. David & Venance, Shannon L. \\
\hline Marshall, Shawn & Nadeau, Yannick & Rathbone, Michel & Stead, John & Viswanathan, Anand \\
\hline Martin, Wayne & Nance, Martha & Redekop, Gary J. & Stefanelli, Mark & Wang, Chun-Bo \\
\hline Masellis, Mario & $\mathrm{Ng}$, Kuan H. & Resch, Lothar & Stemmer-Rachamimov, A. & Warren, Sharon \\
\hline Massoud, Fadi & Nguyen, Dang K. & Richer, Lawrence P. & Steven, David A. & Weber, Frank \\
\hline Mazzini, Letizia & Norris, John & Rosa-Neto, Pedro & St Louis, Erik K. & Weinshenker, Brian \\
\hline McCready, Elizabeth & Norton, Jonathan A. & Rouleau, Guy A. & Stockler-Ipsiroglu, Sylvia & West, Michael \\
\hline McDonald, Patrick & O'Connell, Megan & Rouleau, Isabelle & Stolz, Erwin & Wheatley, Matt B. \\
\hline McKelvey, Roger & O'Kelly, Cian J. & Rowland, Lewis P. & Stotts, Grant & White, Chris \\
\hline McKeown, Martin J. & Oger, Joel & Sadikovic, Bekim & Struffert, Tobias & Whitehead, Shawn \\
\hline Megyesi, Joseph & Parent, Andre & Sarnat, Harvey B. & Symons, Sean & Wirrell, Elaine \\
\hline Mehta, Vivek & Parker, Vivien & Sarty, Gordon & Tai, Peter & Wolfson, Christina \\
\hline Mendelsohn, Daniel & Pasternak, Stephen & Savard, Martin & Tam, Emily & Wong, John \\
\hline Menon, Bijoy K. & Paterson, Phyllis G. & Schmidt, Matthias & Tampieri, Donatella & Woulfe, John \\
\hline Mestre, Tiago & Paulseth, J.E. Rick & Schondorf, Ronald & Tarnopolsky, Mark & Yager, Jerome \\
\hline Mezei, Michelle M. & Peddareddygari, L.R. & Schreiber, Rick & Tellez-Zenteno, Jose F. & Yao, Jun \\
\hline Mikulis, David J. & Perry, James & Sega, Sasa & ten Hove, Martin & Yavin, Daniel \\
\hline Miller, Tom A. & Pfeffer, Gerald & Selby, Kathryn & Thompson, Charlie & Yip, Stephen \\
\hline Mineyko, Aleksandra & Pokrupa, R. & Seshia, Shashi S. & Tie, Qian & Yong, Keir \\
\hline Mitha, Alim & Pollock, Bruce E. & Shamji, Mohammed F. & Tomandl, Bernd F. & Yu, Eugene \\
\hline Moeller, Jeremy J. & Postuma, Ron & Shankar, Jai Jai Shiva & Toth, Cory & Yuan, Chujun \\
\hline Muir, Keith & Pryse-Philllips, William & Silver, Ian & Vajsar, Jiri & \\
\hline Munhoz, Renato P. & Purdy, Allan & Sinclair, John & Vanier, Marie & \\
\hline Murray, B.J. & Rahimi, Fariborz & Slow, Elizabeth J. & Van Landeghem, Frank & \\
\hline Murray, T. Jock & Rajajee, Venkatakrishna & Soros, Peter & Van Uum, Stan & \\
\hline
\end{tabular}

\section{Make a difference to your profession and become a CJNS reviewer}

The Canadian Journal of Neurological Sciences (CJNS) is always looking for talented and skilled reviewers to submit timely and high quality reviews of manuscripts. Without these volunteers, the Journal cannot maintain a steady stream of high quality, informational articles relevant to readers.

Reviewing for CJNS supports Canadian clinical neurosciences. Make it your top refereeing priority.

The Benefits of Becoming a Reviewer:

- An annual record of all your refereeing contributions for academic promotional purposes.

- Your name included in the "Thank you to Reviewers" list in the February issue of CJNS.

- Eligibility for the "Distinguished Reviewer of the Year" award, recognizing extensive, timely and high quality reviews.

- Reviewers who regularly contribute to the CJNS are considered for the Editorial Board.

- Credits towards your Maintenance of Certification.

- Serving as a CJNS reviewer supports your journal and Canadian clinical neurosciences. 
